Amyloidosis database containing baseline demographic and clinical data. Data was analysed using a Chi-squared test (GraphPad Prism 7.0).

Results: Between 1990-2000 there were 188 new cases of AA Amyloidosis of which $51(27.1 \%)$ had an underlying diagnosis of rheumatoid arthritis (RA), median age at diagnosis of amyloid was 60 years. $37(19.7 \%)$ had a diagnosis of Juvenile Idiopathic Arthritis (JIA), median age 29 years. Between 2007 and 2017 there were 270 new diagnoses of AA Amyloidosis, of whom 57 had RA (21.1\%), median age 66 years, and $5(1.9 \%)$ had JIA, median age 37 years. Since the widespread use of combination disease-modifying and biologic therapy there has been a significant reduction in the incidence of JIA-associated AA Amyloidosis $(p<0.0001)$. In contrast, the incidence of RA-associated AA Amyloidosis is unchanged $(\mathrm{p}<0.1357)$

Conclusions: Advances in the treatment of JIA have significantly reduced the incidence of AA Amyloidosis in this cohort. In contrast, despite largely the same medication and similar treatment targets, RA continues to be responsible for a substantial proportion of new cases of AA Amyloidosis. One possible explanation for this may be that current assessment of disease activity in RA is sufficient to identify those at risk of joint damage or radiological progression of disease but insufficient for identifying peristent subclinical biochemical inflammation. One approach for reducing this risk may be the routine measure of the AA Amyloid precursor protein, serum Amyloid A protein (SAA) in those with RA, as it is well established that levels of SAA of below $10 \mathrm{mg} / \mathrm{L}$ prevents the accumulation of $A A$ Amyloid in those with inflammatory disease.

[1] Lachmann, H. J., Goodman HJ, Gilbertson JA, Gallimore JR, Sabin CA, Gillmore JD, Hawkins PN (2007). Natural history and outcome in systemic AA amyloidosis. N Engl J Med 356(23): 2361-2371.

Disclosure of Interest: None declared

DOI: 10.1136/annrheumdis-2017-eular.5005

\section{THU0077 ANTI-COLLAGEN TYPE II ANTIBODIES ARE ASSOCIATED WITH AN ACUTE ONSET RHEUMATOID ARTHRITIS PHENOTYPE AND PROGNOSTICATE LOWER DEGREE OF INFLAMMATION}

V.A. Manivel ${ }^{1}$, M. Mullazehi ${ }^{1}$, L. Padyukov ${ }^{2}$, H. Westerlind $^{3}$, L. Klareskog ${ }^{2}$, L. Alfredsson ${ }^{3}, \mathrm{~S}$. Saevarsdottir ${ }^{2}$, J. Rönnelid ${ }^{1} .{ }^{1}$ Immunology genetics and Pathology, Uppsala University, Uppsala; ${ }^{2}$ Rheumatology Unit, Department of Medicine, Karolinska University Hospital and Karolinska Institutet; ${ }^{3}$ Institute of Environmental Medicine, Karolinska Institutet, Stockholm, Sweden

Background: Anti-collagen II antibody (anti-CII) positive RA patients present with early but not persistent signs of inflammation and joint erosions. This early antiCll-dependent phenotype coincides with high anti-Cll levels around the time of RA diagnosis, whereafter anti-CII levels drop. Our previous studies showed that this phenotype is associated with in vitro cytokine production by monocytes, activation of granulocytes, and enhanced chemokine production by monocyte/granulocyte cocultures, stimulated with anti-CII containing immune complexes. These in vitro findings argue that elevated anti-CIl levels at time of RA diagnosis are functionally related to the corresponding acute onset RA phenotype.

Objectives: Our previous comparison done in a small RA cohort $(n=274)$ describe that anti-cyclic citrullinated peptide 2 (anti-CCP2) positive patients have a severe long-term prognosis but anti-Cll positive patients have transient inflammation. In the present study we wanted to extend this in a large RA cohort with clinical follow-up data, and to relate to HLA-DRB1* alleles.

Methods: Anti-CII and anti-CCP2 were measured at baseline in 773 patients from the Swedish Epidemiological Investigations in Rheumatoid Arthritis (EIRA) study with clinical follow-up data from the Swedish Rheumatology Quality (SRQ) registry, and 1476 patients with HLA-DRB1* information. Comparisons were done concerning CRP, ESR, TJC, SJC, DAS28, DAS28CRP, pain-VAS, global-VAS and $\mathrm{HAQ}$ at 8 occasions during 5 years, and association to HLA-DRB1* alleles.

Results: Anti-Cll was detected in 6.6\% (97/1476), and anti-CCP2 in 57.9\% $(855 / 1476)$ of the patients. There was no significant difference in treatment strategy at diagnosis for patients with and without those antibodies. Anti-Cll associated with elevated CRP, ESR, SJC, DAS28 and DAS28CRP at diagnosis and up to six months, whereas anti-CCP2 associated with SJC and DAS28 from 6 months to 5 years, but not earlier. The anti-Cll-associated phenotype was strong, and predominated also in anti-CII/anti-CCP2 double positive patients. Compared to baseline levels, anti-CII was asscociated with improvements in CRP, ESR, SJC, TJC and DAS28 over time, whereas anti-CCP2 was associated with deteriorations in SJC and DAS28 over time, compared to antibody negative patients. Anti-CII positive patients achieved EULAR good or moderate response more often than negative patients whereas the opposite was found for anti-CCP2 positive patients (figure). Anti-Cll was positively associated with HLADRB $1{ }^{*} 01$ and HLADRB $1{ }^{*} 03$, with significant interaction, and double positive individuals had $>14$ times higher mean anti-CII levels than HLA double negatives. Whereas smoking associated with elevated anti-CCP2 levels, smokers has lower anti-CII levels.

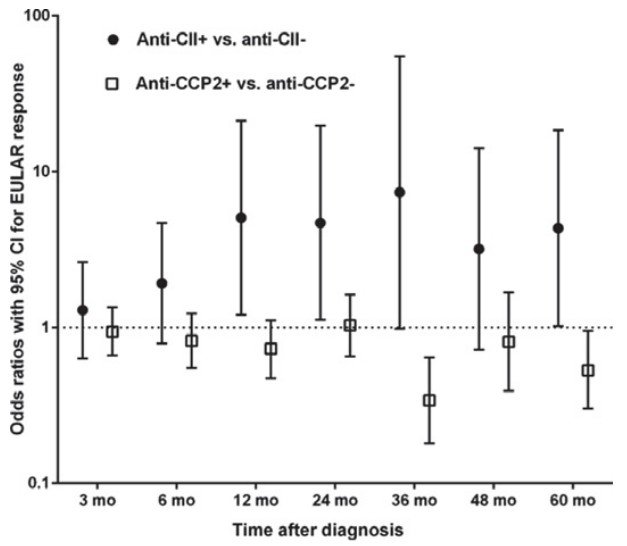

Conclusions: Anti-Cll seropositive RA represents a distinct phenotype, in many respects representing the converse to the clinical, genetic and smoking associations described for ACPA. Although not diagnostically useful, early anti-CII determinations predict favourable inflammatory outcome in RA. The combined analysis of anti-CII and ACPA/anti-CCP2 may be a new two-dimensional tool for predicting the prognosis and chosing therapy in newly diagnosed RA patients.

Disclosure of Interest: None declared

DOI: 10.1136/annrheumdis-2017-eular.2776

\section{THU0078 CONCOMITANT USE OF CONVENTIONAL SYNTHETIC DMARDS AND RESPONSE TO BARICITINIB}

A. Kavanaugh ${ }^{1}$, C. Helt ${ }^{2}$, D. Muram ${ }^{2}$, J. Alam ${ }^{2}$, V. Arora ${ }^{2}$, A.L. Pinto Correia ${ }^{2}$, I. de la Torre ${ }^{2}$, R. van Vollenhoven ${ }^{3}$. ${ }^{1}$ UC San Diego School of Medicine, la Jolla; ${ }^{2}$ Eli Lilly and Company, Indianapolis, United States; ${ }^{3}$ Amsterdam Rheumatology \& Immunol Center, Amsterdam, Netherlands

Background: Baricitinib (BARI), an oral JAK1/JAK2 inhibitor, is in development for patients (pts) with moderate to severe rheumatoid arthritis (RA)..$^{1,2}$

Objectives: This post-hoc analysis of two phase 3 studies assessed whether concomitant use of conventional synthetic disease-modifying antirheumatic drugs (csDMARDs) altered the response or safety outcomes to BARI in RA pts and evaluated the effect of concomitant corticosteroid use on the efficacy of BARI.

Methods: Pts with $\geq 6$ swollen and tender joints and no prior biologic DMARD use were enrolled. In RA-BEAM (NCT01710358), methotrexate (MTX)-inadequate responder (IR) pts were randomised to PBO once daily (QD), BARI $4 \mathrm{mg} Q D$, or adalimumab $40 \mathrm{mg}$ biweekly. ${ }^{1}$ In RA-BUILD (NCT01710358), csDMARD-IR pts were randomised to placebo (PBO) or BARI (2 or $4 \mathrm{mg}) \mathrm{QD}^{2}$ Pts continued background csDMARD (including MTX) therapy. This post-hoc analysis included the PBO $(\mathrm{N}=716)$ and BARI $4 \mathrm{mg}(\mathrm{N}=714)$ pts and assessed the number and type of concomitant csDMARDS and concurrent corticosteroid use.

Results: $71 \%, 21 \%$, and $6 \%$ of PBO pts were taking MTX alone, MTX + $\geq 1$ other csDMARD, and non-MTX csDMARDs, respectively; in BARI $4 \mathrm{mg}$ pts, the

Abstract THU0078 - Table 1. Efficacy and Safety Through 12 Weeks Based on Concomitant csDMARD Usage

\begin{tabular}{|c|c|c|c|c|c|c|}
\hline & \multicolumn{3}{|c|}{ Placebo $(\mathrm{N}=716)$} & \multicolumn{3}{|c|}{ Baricitinib $4 \mathrm{mg}(\mathrm{N}=714)$} \\
\hline & $\begin{array}{c}\text { MTX Alone } \\
(n=506)\end{array}$ & $\begin{array}{c}\text { MTX }+\underset{(n=147)}{\geq 1 \text { csDMARDs }} \\
(n=140\end{array}$ & $\begin{array}{c}\text { Non-MTX csDMARDs } \\
(\mathrm{n}=43)\end{array}$ & $\begin{array}{c}\text { MTX Alone }(n=527) \\
(n=527)\end{array}$ & $\begin{array}{c}\text { MTX }+\underset{(n=131)}{\geq 1 \text { csDMARDs }} \\
(n=1\end{array}$ & $\begin{array}{c}\text { Non-MTX csDMARDs } \\
(\mathrm{n}=42)\end{array}$ \\
\hline \multicolumn{7}{|l|}{ Efficacy measures } \\
\hline ACR20 & $210(42)$ & $57(39)$ & $17(40)$ & $361(69)$ & $85(65)$ & $25(60)$ \\
\hline ACR50 & $84(17)$ & $18(12)$ & $7(16)$ & $227(43)$ & $52(40)$ & $13(31)$ \\
\hline ACR70 & $20(4)$ & $7(5)$ & $3(7)$ & $106(20)$ & $19(15)$ & $5(12)$ \\
\hline SDAI $\leq 11$ & $92(18)$ & $18(12)$ & $10(23)$ & $221(42)$ & $45(34)$ & $14(33)$ \\
\hline SDAI $\leq 3.3$ & $8(2)$ & $3(2)$ & 0 & $52(10)$ & $4(3)$ & $2(5)$ \\
\hline DAS28-ESR $\leq 3.2$ & $11(2)$ & $3(2)$ & $1(2)$ & $58(11)$ & $11(8)$ & $2(5)$ \\
\hline DAS28-ESR $<2.6$ & $35(7)$ & $10(7)$ & $4(9)$ & $129(24)$ & $27(21)$ & $7(17)$ \\
\hline \multicolumn{7}{|l|}{ Safety measures } \\
\hline$\geq 1$ adverse event & $442(87)$ & $132(90)$ & $39(91)$ & $465(88)$ & $116(89)$ & $40(95)$ \\
\hline$\geq 1$ serious adverse event & $14(3)$ & $4(3)$ & $3(7)$ & $14(3)$ & $1(1)$ & $1(2)$ \\
\hline Discontinuation due to adverse event & $16(3)$ & $2(1)$ & 0 & $11(2)$ & $5(4)$ & $3(7)$ \\
\hline Death & $1(0.2)$ & $1(0.7)$ & 0 & 0 & 0 & 0 \\
\hline
\end{tabular}

Data are $\mathrm{n}(\%)$ 
rates were $74 \%, 18 \%$, and $6 \%$, respectively. Oral corticosteroids were used in $56 \%$ of PBO and $55 \%$ of BARI pts at baseline; pts continued use throughout the studies. The differences in clinical efficacy between BARI $4 \mathrm{mg}$ and PBO at 12 weeks was similar regardless of the number or type of csDMARDs concomitantly used (Table) or the concomitant use of corticosteroids (data not shown). The rates of serious adverse events and discontinuation due to adverse events were comparable regardless of the number or type of csDMARDs used (Table) or corticosteroid use.

ACR20/50/70 $=20 \%, 50 \%$, and $70 \%$ improvement in American College of Rheumatology criteria; csDMARDs=conventional synthetic disease-modifying antirheumatic drugs; DAS28-ESR=Disease Activity Score 28-erythrocyte sedimentation rate; $\mathrm{MTX}=$ methotrexate; SDAI=Simple Disease Activity Index.

Conclusions: BARI has demonstrated clinical safety and efficacy in a wide range of pts, regardless of the number of concomitant csDMARDs or concomitant use of corticosteroids.

References:

[1] Taylor PC et al. Arthritis Rheumatol 2015;67(S10):3927-3928.

[2] Dougados M et al. Ann Rheum Dis 2017;76:88-95.

Disclosure of Interest: A. Kavanaugh Consultant for: Eli Lilly and Company, C. Helt Employee of: Eli Lilly and Company, D. Muram Employee of: Eli Lilly and Company, J. Alam Employee of: Eli Lilly and Company, V. Arora Employee of: Eli Lilly and Company, A. L. Pinto Correia Employee of: Eli Lilly and Company, I. de la Torre Employee of: Eli Lilly and Company, R. van Vollenhoven Grant/research support from: Abbvie, Amgen, BMS, GSK, Pfizer, Roche, UCB, Consultant for: Abbvie, Biotest, BMS, Celgene, Crescendo, GSK, Janssen, Eli Lilly and Company, Merck, Novartis, Pfizer, Roche, UCB, Vertex

DOI: 10.1136/annrheumdis-2017-eular.1342

\section{THU0079 EVALUATION OF SYNOVITIS IN THE FOOT AND THE ANKLE NOT INCLUDED IN THE 28-JOINT DISEASE ACTIVITY SCORE USING ULTRASONOGRAPHY AND HISTOLOGICAL FINDINGS IN THE PATIENTS WITH RHEUMATOID ARTHRITIS}

\section{A. Abe, H. Ishikawa. Rheumatology, Niigata Rheumatic Center, Shibata, Japan}

Background: In the treatment of rheumatoid arthritis (RA), the early diagnosis and early medical treatment via tight control have become increasingly important with the advent of biological therapy. Ultrasonography (US) for the affected joints enables the evaluation of synovial hypertrophy, effusion and bone erosion in real time. US is a reliable method that can detect more erosive sites than radiography. US is now utilized widely and is a reliable tool of rheumatologists for diagnosing RA and evaluating the disease activity.

Objectives: This study was conducted to clarify the relationship between the systemic disease activity, local disease activity using US and a synovial histopathological evaluation.

Methods: Between March 2011 and September 2015, 668 joints were surgically treated on the whole, and synovial biopsies were performed at the time of surgery. Among them, a total of 118 toes and 21 ankles, which are not included in the 28-joint disease activity score (DAS28), were investigated. Just before surgery, the US probe was placed on the dorsal aspect of the ankle and/or the toes to evaluate the activity of local synovitis. The maximum grade of power Doppler (PD) signal was determined, ranging from 0 to 3 . The serum $C$ reactive protein (CRP), matrix metalloproteinase-3 (MMP-3) and DAS28 values were also examined

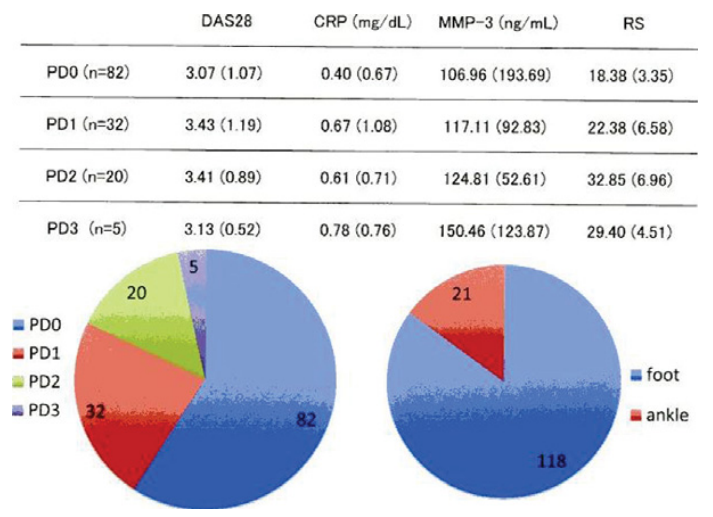

just before surgery. A histopathological examination of the gathered synovium at the surgical site was performed using the Rooney score (RS). Biological disease-modifying antirheumatic drugs were used in 45 cases, namely infliximab in 7 cases, etanercept in 16, adalimumab in 7 , tocilizumab in 6 , abatacept in 4 , certolizumab pegol in 1, and golimumab in 4 .

Results: The PD score was grade 0 in 82 cases, 1 in 32 cases, 2 in 20 cases and 3 in 5 cases. The total RS and its item scores except for "proliferating blood vessels" correlated well with the PD signal intensity. The systemic disease activity, as indicated by DAS28, CRP and MMP-3, had no significant correlation with the local PD signal intensity. However, the DAS28, CRP and MMP-3 values and each RS item score, except for "proliferating blood vessels", were significantly lower in the PD grade 0 group than in the PD grades 1, 2 and 3 groups.

Conclusions: No PD signal intensity in the ankle and foot indicates systemically well-controlled disease activity. US is an excellent tool for determining local synovitis as well as the systemic disease activity in patients with RA

Disclosure of Interest: None declared

DOI: 10.1136/annrheumdis-2017-eular.2011

\section{THU0080 DEPRESSION AND ANXIETY REDUCE THE LIKELIHOOD OF ACHIEVING REMISSION IN PATIENTS WITH RHEUMATOID ARTHRITIS: REAL LIFE DATA FROM THE NOR-DMARD STUDY}

B. Michelsen $^{1,2}$, E.K. Kristianslund ${ }^{2}$, K.M. Fageli ${ }^{2}$, E. Lie ${ }^{2}$, H.B. Hammer ${ }^{2}$, G. Haugeberg ${ }^{1,3}$, T.K. Kvien ${ }^{2} .{ }^{1}$ Dept. of Rheumatology, Hospital of Southern Norway Trust, Kristiansand; ${ }^{2}$ Dept. of Rheumatology, Diakonhjemmet Hospital, Oslo; ${ }^{3}$ Dept. of Rheumatology, Martina Hansens Hospital, Bærum, Norway

Background: Depression and anxiety are reported to predict poorer treatment outcomes in rheumatoid arthritis (RA). ${ }^{1}$ Whether this can be confirmed in larger prospective observational studies using various remission criteria remains to be explored.

Objectives: To investigate the predictive value of baseline depression/anxiety on the likelihood of achieving remission in RA as well as the associations between baseline depression/anxiety and the components of the remission criteria at follow-up.

Methods: From the prospective, multi-center NOR-DMARD study we included RA patients starting first-time tumour necrosis factor inhibitors (TNFi) and DMARD naïve RA patients starting methotrexate (MTX) between year 2006 and 2012. The following two criteria for depression/anxiety were assessed: 1) the Medical Outcomes Survey Short Form-36 (SF-36) Mental Health subscale (MH) $\leq 56$ and 2) the SF-36 Mental Component Summary score (MCS) $\leq 38 .^{2}$ The predictive value of baseline depression/anxiety on remission after 3 and 6 months treatment was explored in prespecified logistic regression models adjusted for age, sex, disease duration and smoking and the associations between baseline depression/anxiety and the different components of the remission criteria at follow-up in prespecified multiple linear regression models adjusted for age, sex, disease duration and smoking. Results: A total of 1450 RA patients were included (mean (SD) age 54.4 (13.5) years, median (25th-75th percentile) disease duration $0.4(0.0-5.0)$ years, $68.7 \%$ females and $28.6 \%$ current smokers). According to the SF- $36 \mathrm{MH} \leq 56 / \mathrm{SF}-$ $36 \mathrm{MCS} \leq 38$ criteria $18.1 / 29.9 \%$ of the patients were depressed/anxious at baseline, respectively. Lower percentages of patients with versus without baseline depression/anxiety achieved remission at 3 and 6 months treatment (unadjusted analyses, figure). Baseline depression/anxiety negatively predicted remission after 3 and 6 months (adjusted analyses, table 1 and 2 ).

The findings were confirmed in separate subgroup analyses of TNFi/MTX treated patients. Baseline depression/anxiety were associated with increased patient's and evaluator's global assessment and 28 tender joint count at 3 and 6 months, but not with level of acute phase reactants or 28 swollen joint count.

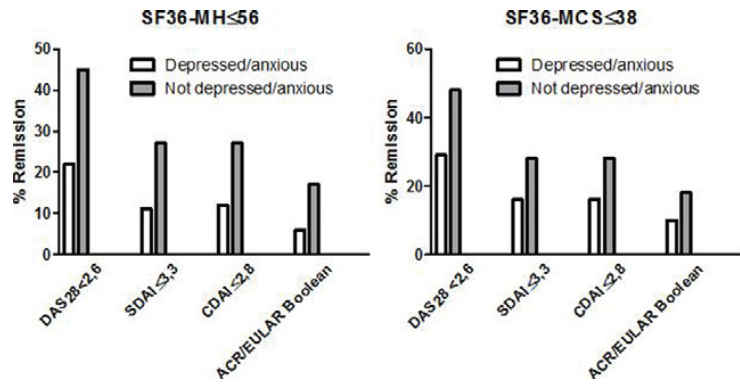

Abstract THU0080 - Table 1. Odds ratio $(95 \% \mathrm{Cl})$ for remission in patients with versus without baseline depression/anxiety according to the SF36-MH $\leq 56$ criterion

\begin{tabular}{|c|c|c|c|c|}
\hline Months & DAS28<2.6 & $\mathrm{SDAI} \leq 3.3$ & $\mathrm{CDAI} \leq 2.8$ & ACR/EULAR Boolean \\
\hline 3 & $0.48(0.32-0.73), p<0.001$ & $0.43(0.25-0.74), p=0.003$ & $0.38(0.22-0.65), p<0.001$ & $0.27(0.13-0.57), p=0.001$ \\
\hline 6 & $0.36(0.24-0.56), p<0.001$ & $0.35(0.20-0.60), p<0.001$ & $0.38(0.23-0.63), p<0.001$ & $0.31(0.16-0.61), p=0.001$ \\
\hline
\end{tabular}

Abstract THU0080 - Table 2. Odds ratio $(95 \% \mathrm{Cl})$ for remission in patients with versus without baseline depression/anxiety according to the SF36MCS $\leq 38$ criterion

\begin{tabular}{lcccc}
\hline Months & DAS28ESR $<2.6$ & SDAI $\leq 3.3$ & CDAI $\leq 2.8$ & ACR/EULAR Boolean \\
\hline 3 & $0.67(0.49-0.93), p=0.02$ & $0.51(0.34-0.78), p=0.002$ & $0.47(0.32-0.70), p<0.001$ & $0.35(0.21-0.59), p<0.001$ \\
6 & $0.45(0.32-0.63), p<0.001$ & $0.46(0.31-0.69), p<0.001$ & $0.48(0.33-0.69), p<0.001$ & $0.46(0.29-0.72), p=0.001$
\end{tabular}

